\title{
PROYECTO DE CATALOGACION RETROSPECTIVA EN LA UNIVERSIDAD CARLOS III
}

\section{Bustelo Ruesta *, P. Alonso Rodríguez"* y M. Taladriz Mas**}

Resumen: La Biblioteca de la UCAR III, debido a las circunstancias de su creación y rápido desarrollo, ha tenido que afrontar un proyecto de catalogación retrospectiva. Utilizando los servicios de la empresa Gabinete de Asesores Documentalistas, S. A., el proceso se abordó con una fase de preparación inicial que incluía la clasificación e indización para la realización de una estadística previa y la redacción del manual de procedimiento. Se utilizaron diversas modalidades de proceso. Una de ellas fue la importación de registros de Bibliofile y de Bibliografía Española, obteniendo muy distintos resultados según la materia de la que trate el fondo.

Palabras clave: Catalogación restrospectiva, UCAR III, captación de registros, Bibliofile, Bibliografia Española.

\begin{abstract}
The UCAR III Library, because of the circumstances of its creation and fast development, has had to face a retrospective cataloguing project. Using the services of Gabinete de Asesores Documentalistas, S. A., the process began with an initial preparatory phase that includes book's classification and indexation, a previous statistics and the writing of the proceeding handbook. Different processing options were used. One of them was the capture of bibliographic records from Bibliofile and $\mathrm{Bi}$ bliografia Española, obtaining very different results depending on the subject of the collection.

Keywords: Retrospective cataloguing, UCAR III, capture of bibliographic records, Bibliofile, Bibliografia Española.
\end{abstract}

\section{Introducción}

La Biblioteca de la UCAR III, con sólo 4 años de existencia, ya ha tenido que afrontar 2 reconversiones, aunque a pequeña escala comparadas con otras que han tenido que llevar a cabo bibliotecas de una mayor entidad.

La UCAR III es una universidad de reciente creación, éste es su cuarto año, estructurada en 2 campus, uno de Ciencias Sociales y una Escuela Politécnica. El Servicio de Biblioteca surge como un servicio centralizado en cada uno de los campus, con una coordinación y dirección única, así como un presupuesto centralizado.

El mayor problema que afronta una biblioteca que comienza «ex novo» es la formación de la colección. El tiempo corre deprisa, se suceden los cursos acadé-

* Gabinete de Asesores Documentalistas, S. A.

** Universidad Carlos III.

Recibido: 20-8-93 
micos y se plantean serias dificultades para poner a disposición de los usuarios la bibliografia que precisan para llevar a cabo sus trabajos de estudio o investigación.

Tres factores abonaron las dificultades reseñadas más arriba:

- El gran volumen de material que es necesario adquirir.

- La escasez de personal.

- Los problemas informáticos.

Como consecuencia de una dotación inicial por parte del Ministerio de Educación, se disponía de un presupuesto importante, 140.000 .000 de ptas., para la adquisición de material bibliográfico. La actividad académica comenzó con anterioridad a la puesta en marcha del incipiente servicio de biblioteca. El ritmo de adquisición era frenético, apoyado por la urgencia del personal docente y los alumnos en conseguir la bibliografia necesaria.

La fórmula de adquisición, petición y recepción fue poco ortodoxa en cuanto al control de pedidos e incluso la selección de proveedores. El control de adquisiciones era absolutamente manual. En abril de 1990 se empieza a barajar la posibilidad de empezar a automatizar la catalogación.

Habiendo iniciado las actividades la biblioteca en octubre de 1989 (sólo 6 meses antes), ya se disponía de un conjunto de 3.600 registros bibliográficos en ficha, que se entregaron a una empresa de servicios para su conversión en formato MARC. Eran fichas normalizadas y con las autoridades controladas.

La puesta en marcha del módulo de catalogación ABSYS supuso un parón en el ritmo de catalogación manual que se iba desarrollando. Los técnicos de catalogación tenían que adaptarse al nuevo procedimiento y el sistema informático daba problemas de capacidad de la máquina.

Así llega el traslado a un nuevo edificio de biblioteca, que lleva a una nueva ralentización del proceso automatizado y que supuso la pérdida de $725 \mathrm{~h}$. de catalogación (instalación de la red, cambio de un 386 a un 486, adquisición de terminales suficientes para proceso, etc.) aunque no se desaprovecha el tiempo y con la espléndida colaboración de becarios de los departamentos de Derecho y Economía, personal contratado temporalmente y personal en prácticas de los I.F.P., rama de Biblioteconomía, se acometen diferentes tareas:

- Becarios de Departamentos:

* Revisión del fichero de autoridades.

* Clasificación del fondo bibliográfico pendiente de proceso, bajo la coordinación de los técnicos de la biblioteca.

- Alumnos en prácticas de Formación Profesional.

* Recuperación de registros en lengua inglesa en las áreas de Economía, Empresa y Biblioteconomía, a partir de la base de datos Bibliofile, registros que luego serán incorporados en el "pool bibliográfico» de nuestro sistema.

- Personal contratado.

* Puesta al día de listados de adquisiciones y verificación de pedidos recibidos, agotados, etc., para poder introducir dichos datos en el módulo de adquisiciones que se iba a poner en marcha en julio de 1991. El trabajo de introducción de datos lo lleva a cabo una empresa grabadora de datos. 
Al tiempo se cargan las cintas con los registros de la Biblioteca Nacional correspondientes a las áreas de Historia, Derecho, Filosofia, Administración Pública e Informática, en un total de 97.460 registros.

Como consecuencia de todos los tropiezos mencionados anteriormente, se fue acumulando un fondo cercano a los 7.000 volúmenes proveniente sólo de adquisiciones, sin contar los numerosos donativos que urgía poner en servicio, y urgía porque era un fondo muy demandado por los usuarios y si no se conseguía peligraba el proyecto de biblioteca centralizada. Ya se empezaba a esgrimir el argumento de "este sistema no funciona, no son capaces de sacar los libros a las estanterías en tiempo" y curiosamente ese número citado anteriormente de libros pendientes de proceso crecía día a día en el lenguaje de los usuarios y se contaban historias de grandes cantidades de libros almacenados en los sótanos profundos.

Después de haber llevado a cabo algunos estudios sobre la posibilidad de afrontar el proceso de ese fondo pendiente con nuestros propios recursos humanos, se vio que era inviable y se decidió contratar su proceso (exclusivamente la descripción) con una empresa de servicios (Gabinete de Asesores Documentalistas).

\section{Diseño del proyecto}

La primera vez que la Biblioteca de la Universidad se pone en contacto con Gabinete de Asesores Documentalistas para estudiar la posibilidad de llevar a cabo este proyecto, ya se habían establecido unos principios previos:

- Se trataba de recuperar toda la información posible de las bases de datos de Bibliofile y Bibliografia Española. Los registros recuperados de Bibliofile por los alumnos en prácticas y los registros de la Biblioteca Nacional se encontraban cargados en el "pool bibliográfico» sobre el que se debía trabajar.

- Se debía aprovechar la precatalogación que se había realizado de gran parte del fondo.

- El fondo a catalogar ya había sido indizado y clasificado previamente por becarios de departamentos coordinados por los técnicos de la Biblioteca, quedando reflejados los descriptores y notaciones en el interior de los libros.

- El trabajo debía realizarse directamente en las dependencias y sistema informático de la Biblioteca de la Universidad, lo que permitiría no trasladar los fondos y que el trabajo que se fuera realizando estuviera consultable en el mismo momento.

Sin embargo, quedaba por definir exactamente la casuística que podía presentar el fondo y tratar de aproximar los porcentajes de cada modalidad, para elaborar un presupuesto realista del que dependía la viabilidad del proyecto.

Para ello, GAD realizó un muestreo previo entre el fondo a catalogar. Se eligieron 231 monografias que correspondían tanto a Derecho como a Economía y a distintos períodos de entrada en la biblioteca, ya que los libros se encontraban ordenados en las estanterías por su orden de llegada en las dos grandes divisiones mencionadas. 
Como resultado de este muestreo se pudo obtener:

- La definición exacta de las distintas modalidades de proceso y de cada una de la tareas a realizar en cada caso (reflejadas en el cuadro A).

- La estadística de los porcentajes estimados en cada una de las modalidades.

En resumen, los resultados fueron los siguientes:

* Catalogaciones ya existentes en la base de datos de la biblioteca. (Duplicados, otras ediciones o volúmenes de una colección.) (Modalidades A, C, E y F) ............................ 14\%

* Catalogaciones recuperables de otras fuentes. (Bibliofile y Bibliografia Española.)

(Modalidad B) ........................................ 16\%

* Precatalogaciones existentes en la base de datos de la biblioteca. (Modalidad D) ........................................ 56\%

* Catalogaciones originales.

(Modalidad G) ......................................... 14\%

\section{Desarrollo del proyecto}

\section{a) Redacción del manual de procedimiento}

Antes de comenzar el trabajo, técnicos de la biblioteca y de GAD redactaron conjuntamente el manual de procedimiento.

En él se especificaba la manera de introducir los datos en cada una de las etiquetas del formato IBERMARC, la utilización de reglas ortográficas (mayúsculas, acentos, abreviaturas, iniciales, etc.), la forma de consignar la puntuación ISBD y la forma de consulta y establecimiento de los campos de autoridades.

Este manual evitó que durante el desarrollo del proyecto se produjeran vacilaciones o cambios de criterio, resultando un instrumento de enorme utilidad y consulta obligada durante el proceso.

\section{b) Medios utilizados}

El proyecto se llevó a cabo en un período de tres meses, finalizando con el proceso de un total de 6.361 monografias.

El equipo de trabajo se formó con tres documentalistas, un auxiliar documentalista y un jefe de equipo, todos ellos pertenecientes a la plantilla de GAD.

El auxiliar tuvo a su cargo exclusivamente el tratamiento de las modalidades A y B que se reflejan en el cuatro anterior (detección de duplicados y captación de registros de otras fuentes). Los documentalistas realizaban fundamentalmente el proceso de las restantes modalidades, aunque también intervinieron en la modificación y corrección de los registros importados. El jefe de equipo se encargó de la revisión, corrección y unificación de criterios de todas las catalogaciones.

La biblioteca de la universidad puso a disposición de GAD 3 terminales (durante todo el horario de apertura, 8 a.m. a 10 p.m.) conectados a su ordenador 

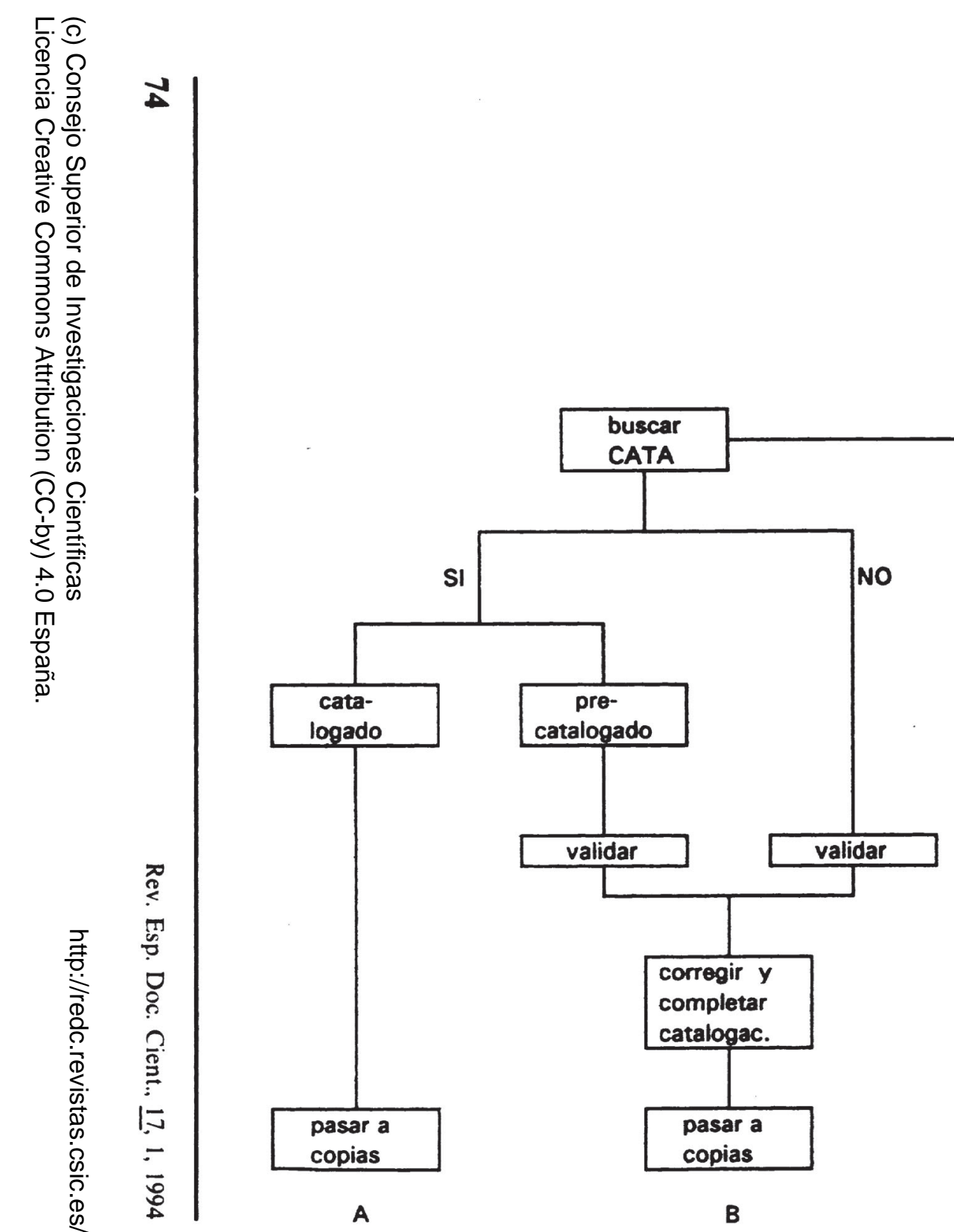

Condro A

B

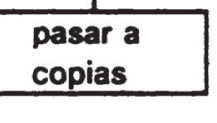

c

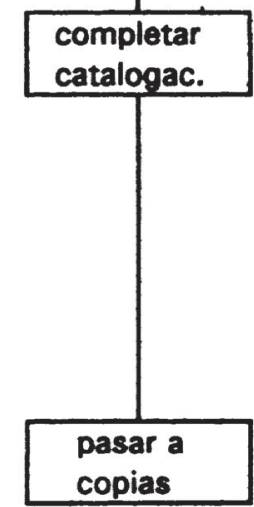

D buscar

SI
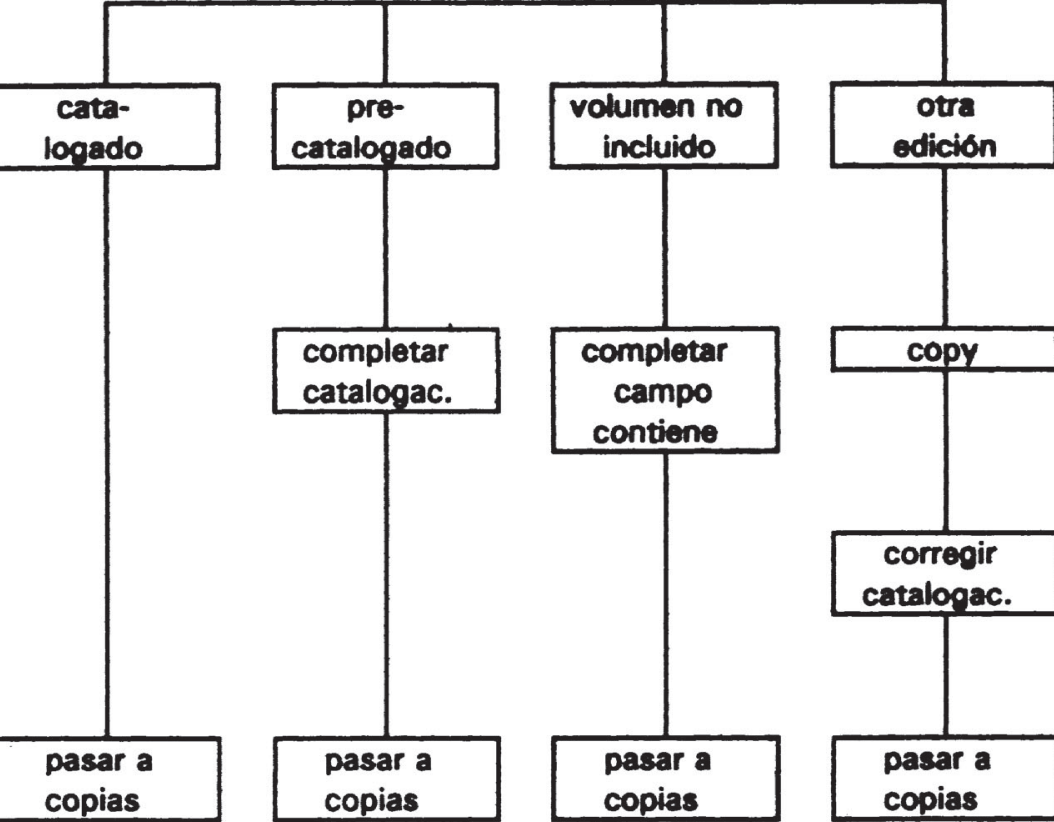

copy

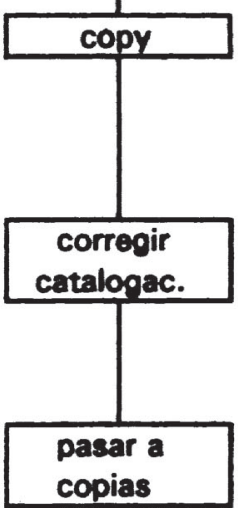

E
F 
central y con claves de usuario para todas las funciones del programa de gestión ABSYS.

c) Resultados cuantitativos

El total del fondo procesado puede dividirse en las siguientes especialidades o secciones:

- Derecho (4.422).

- Economía (Fondo de Adquisiciones, 1.227).

- Economía (Donación Arnaiz, 482).

- Fonoteca (38).

- Biblioteconomía (151).

- Informática (41).

Los resultados generales de todo el fondo procesado difieren en algunos puntos con la estadística realizada previamente (catalogaciones ya existentes: $9,2 \%$, catalogaciones recuperadas de otras fuentes: $20,4 \%$, precatalogaciones: $51,7 \%$ y catalogaciones originales: $18,7 \%$ ), como puede comprobarse en el cuadro B. Esta diferencia puede haberse producido por la introducción de fondos no contemplados inicialmente (Fonoteca, Biblioteconomía, Informática y Donación Arnaiz). Aún así los porcentajes son muy aproximados, por lo que la estadística previa cumplió perfectamente sus objetivos, a pesar de realizarse sobre un pequeño porcentaje del total $(3,5 \%$ del fondo).

\section{Cuadro B}

\begin{tabular}{lcr}
\hline & Previsto & Real \\
\cline { 2 - 3 } & $14 \%$ & $9,2 \%$ \\
Catalogaciones ya existentes en la base de datos & $16 \%$ & $20,6 \%$ \\
Catalogaciones recuperadas de otras fuentes & $56 \%$ & $51,5 \%$ \\
Precatalogaciones ya existentes & $14 \%$ & $18,7 \%$ \\
Catalogaciones originales & \\
\hline
\end{tabular}

Además de los resultados generales, resulta muy interesante desglosar por las distintas materias lo que se ha podido recuperar de las fuentes externas. Estos resultados se recogen en el cuadro $C$.

\section{Cuadro C}

Distribución de catalogaciones recuperadas en otras fuentes

\begin{tabular}{lccccccc}
\hline & N. Libros Rec. bibliofile & $\%$ & Recup. BN & $\%$ & Total rec. & $\%$ \\
\cline { 2 - 7 } & 4.422 & 28 & 0,6 & 735 & 16,6 & 763 & 17,2 \\
Derecho & 1.227 & 516 & 42 & 18 & 1,5 & 534 & 43,5 \\
Econ. Fondo Moderno & 482 & 0 & 0 & 0 & 0 & 0 & 0 \\
Econ. Donación Arnáiz & 38 & 0 & 0 & 1 & 2,6 & 1 & 2,6 \\
Fonoteca & 151 & 1 & 0,7 & 11 & 7,3 & 12 & 8 \\
Biblioteconomía & 41 & 4 & 9,8 & 1 & 2,4 & 5 & 12,2 \\
Informática & 6.361 & 549 & 8,6 & 766 & 12 & 1.315 & 20,6 \\
Total & & & &
\end{tabular}


Como vemos, el fondo moderno de Economía da muy buenos resultados de recuperación en Bibliofile ( $42 \%$ ), mientras que el fondo de Derecho obtiene mucho mejores resultados en Bibliografia Española $(16,6 \%)$.

En este punto hay que resaltar que en escasas ocasiones se dio la coincidencia de encontrar la misma catalogación en las dos fuentes. Cuando esto ocurrió, se prefirió la catalogación de Bibliofile por las razones que más adelante expondremos.

\section{d) Caracteristicas del trabajo realizado}

En las catalogaciones ya existentes en la base de datos, el trabajo consistió en añadir los datos necesarios cuando se trataba de volúmenes que completaban una colección o en copiar el registro cambiando el número de la edición y el año cuando se trataba de otra edición. También establecimos el criterio de verificar que los descriptores y la CDU que se encontraban en el interior del libro coincidian con los existentes en los registros de la base de datos. En un tanto por ciento elevado coincidían plenamente. Cuando no era así, se añadía el descriptor propuesto en el campo correspondiente. En caso de absoluta discrepancia se pasaba a consideración de la responsable de la Sección de Proceso.

En los registros captados de otras fuentes, la labor consistió en revisar la catalogación, adaptar los encabezamientos a los utilizados en la Biblioteca y añadir los datos de descriptores y CDU. En este tipo de registros, hay que destacar que resultaban más aprovechables los que provenían de Bibliofile que los de Bibliografia Española. Aunque en los registros de Bibliofile había que cambiar muchos encabezamientos de entidades, notas y abreviaturas debido a la diferencia de idioma, y la puntuación de algunos campos, el balance final es más positivo que el de los registros españoles. En los registros de BE encontramos campos 008 defectuosos, gran profusión de títulos uniformes innecesarios, algunos defectos de utilización de los campos de encabezamientos principales y secundarios y algunos problemas de caracteres con las letras acentuadas y las eñes. Esto supuso un nivel de corrección importante que no siempre podía llevar a cabo el auxiliar sin ayuda.

También habria que destacar que muchas veces encontrábamos distintas catalogaciones procedentes de BE para un solo registro. Entre ellas tenían algunas diferencias que obligaban a sopesar cuál elegir, retrasando un poco el proceso.

Entre las precatalogaciones existían grandes diferencias. Las provenientes del departamento de adquisiciones, realizadas a la hora de hacer la petición del libro, eran bastante completas y servían de base para completar la catalogación. Sin embargo, las que pertenecían a la grabación masiva de fichas manuales tenían una calidad que dejaba mucho que desear. En la mayor parte de los casos, había que rehacer completamente la catalogación, como si se tratase de una catalogación original.

En las catalogaciones originales se siguieron los criterios establecidos por la Biblioteca, consultando cada una de las autoridades que se incluían en la base de datos.

Por último, habría que destacar la gran facilidad de manejo que tiene la función de integración de registros del programa ABSYS, lo cual supuso una gran ventaja 
para el desarrollo del proyecto, sobre todo en las opciones de captación de registros de fuentes extrenas.

\section{Conclusiones}

- El trabajo previo a la catalogación retrospectiva (búsquedas en Bibliofile, asignación de descriptores y clasificación, y redacción del manual de procedimiento) tuvo una gran importacia a la hora de aumentar la eficacia del proceso. Por nuestra experiencia, consideramos que una buena fase de preparación del trabajo de conversión retrospectiva es aconsejable siempre y que se traduce en logros de mayor rentabilidad y eficacia.

- Cuando existen distintas modalidades de proceso, un muestreo significativo sobre un pequeño porcentaje del fondo es suficiente para realizar una estadística previa que nos permita ajustar los presupuestos y tomar decisiones sobre el procedimiento a seguir.

- Tratándose de fondos de reciente adquisición (si exceptuamos los 482 libros de la Donación Arnaiz), nos sorprende el bajo porcentaje de recuperaciones en Bibliofile y Bibliografia Española que hemos obtenido. Aún así, existen grandes diferencias entre los dos grandes bloques (Derecho y Economia), en los que el número de libros procesados es suficiente para poder extrapolar las cifras. Claramente cuando tratamos fondos habituales de los requeridos en una facultad de Económicas, la fuente a utilizar es Bibliofile, siendo para los fondos de una facultad de Derecho mucho más eficaz Bibliografia Española.

En cualquier caso, los resultados globales nos indican que cualquier proyecto de catalogación retrospectiva no se debe basar únicamente en la recuperación de catalogaciones de fuentes externas. En nuestro caso, esta modalidad ha cubierto sólo un $20 \%$ del total.

- Por último, un comentario sobre la utilidad de las precatalogaciones en un proceso de conversión retrospectiva. La utilidad está directamente relacionada con la calidad de dichas precatalogaciones, siendo de gran importancia no tener que volver a entrar en los campos ya cumplimentados en la fase de precatalogación. En caso contrario, las precatalogaciones no suponen ninguna ayuda con respecto a la catalogación original, sino muchas veces todos lo contrario. 